\title{
LIDERAZGO DE LOS DOCENTES DE FORMACIÓN PROFESIONAL BÁSICA PARA LA MEDIACIÓN ESCUELA-EMPLEO DEL ALUMNADO CON DIVERSIDAD FUNCIONAL INTELECTUAL
}

\author{
María Isabel Negri Cortés \\ Universidad Internacional de La Rioja \\ Juan José Leiva Olivencia \\ Universidad de Málaga
}

RESUMEN: Los ciclos de Formación Profesional Básica son una plataforma emergente para la formación e inserción sociolaboral de jóvenes con Diversidad Funcional Intelectual teniendo el profesorado un papel fundamental. En este sentido, el objetivo de este trabajo fue describir y analizar el liderazgo que desempeñan los docentes al actuar como dinamizadores de los procesos de mediación entre los contextos escolares y laborales.

Se ha contado con una muestra incidental de 38 docentes de institutos de educación secundaria de Málaga que ofertan ciclos de Formación Profesional Básica. Los datos se han recogido a través de un cuestionario adhoc valorado por un grupo de expertos junto con cinco entrevistas en profundidad a los profesionales de uno de estos centros.

Se ha aplicado análisis cualitativo a las entrevistas, así como un análisis estadístico descriptivo a los datos del cuestionario. Los resultados muestran la necesidad de asumir por parte de los docentes un liderazgo compartido que capacite al alumnado con Diversidad Funcional Intelectual en habilidades y competencias socio-personales que faciliten su acceso al empleo, y actuando como mediadores entre el ámbito formativo y laboral, concluyendo en la necesidad de potenciar una adecuada capacitación docente y de promover la flexibilización curricular y organizativa del contexto educativo.

PALABRAS CLAVE: Liderazgo docente, mediación laboral, discapacidad intelectual, formación profesional. 


\title{
TEACHERS LEADERSHIP IN BASIC VOCATIONAL TRAINING FOR MEDIATION BETWEEN SCHOOL AND EMPLOYMENT OF THE STUDENTS WITH INTELLECTUAL FUNCTIONAL DIVERSITY
}

\begin{abstract}
The Basic vocational training programs are a platform to the training and social and labor access of youngs with intellectual functional diversity, where the teachers have an essential role. The objective of our paper was to describe and analyze the leadership of the teacher when they act like mediators between scholar and labor contexts.

We had an incidental sample made of 38 teachers of secondary education school of Málaga. The data were collected by an adhoc questionnaire that was valued for an expert group, and also by five depth interview to the participants of one of these schools.

We applied the qualitative analysis to the interviews and the descriptive statistical analysis to the questionnaire data. The results point that is necessary the teachers work through shared leadership for teach habilities and skill that help to get a job to the students with intellectual functional diversity, being mediators between the training and social contexts. Like conclusion, is necessary to improve the initial training of the teachers and get better the curricular flexibilization and organization of the school.
\end{abstract}

KEYWORDS: Teacher leadership, labor mediation, intellectual disability, vocational training.

Recibido: 30/11/2018

Aceptado: 25/06/2019

Correspondencia: Juan José Leiva Olivencia, Universidad de Málaga, Bulevar Louis Pasteur, 25, Campus de Teatinos, 29010-Málaga. Email: jjleiva@uma.es.

\section{INTRODUCCIÓN}

\subsection{Los ciclos de Formación Profesional Básica como estrategias de capacitación para el empleo de los jóvenes con DFI}

Para alcanzar el objetivo de la plena inclusión de las personas con Diversidad Funcional Intelectual (en adelante DFI) en la vida adulta, resulta fundamental que estas puedan tener acceso a los entornos económicos y laborales que garantizarán su independencia social, de acuerdo con los principios de equidad y justicia social. En este sentido, debemos ser conscientes de las dificultades a las que debe enfrentarse este colectivo para superar su inserción laboral en el mercado de trabajo ordinario, debido generalmente a su tradicional exclusión de este y otros contextos de la vida adulta, y que actualmente son eje central de las inquietudes de los profesionales educativos, así como del alumnado con DFI y sus familias. 
Autores como García-Gómez, Ordoñez-Sierra y Izquierdo (2018), en sus investigaciones acerca del rol que desempeñan las familias del alumnado de formación profesional, destacan una intencionalidad clara de estas hacia el acceso al empleo de sus hijos e hijas, en relación a su preocupación hacia un futuro profesional estable y seguro. Podemos deducir por tanto, que en el caso de las familias de jóvenes con DFI, esta preocupación puede resultar aún mayor, dados los prejuicios que existen hoy en día hacia estos jóvenes en el mercado de trabajo.

Como estrategia preventiva de una posible exclusión social, debemos tener en cuenta que, para que estos jóvenes obtengan satisfactoriamente un puesto trabajo, es imprescindible que reciban una formación específica que se ajuste a sus necesidades personales, y que les ayudará a mejorar sus condiciones de empleabilidad y a incrementar sus oportunidades de participación laboral (Fundación ONCE, 2015). En este sentido, debemos considerar la existencia de varias investigaciones como la de Vilà, Pallisera y Fullana (2010) y la de Negri y Leiva (2017), que determinan que los aprendizajes adquiridos durante las etapas educativas previas a la inserción laboral pueden erigirse como un factor clave para favorecer las posibilidades de los jóvenes con DFI de desarrollar una actividad profesional en el mercado laboral ordinario.

En relación a esta idea, podemos hablar del posible impacto que los itinerarios de formación profesional pueden generar hacia el éxito profesional del colectivo al que nos referimos. Para atender a la capacitación en habilidades y competencias de índole laboral de los jóvenes con DFI, desde la publicación de la LOGSE (1990) se ofertan los Programas de Garantía Social, que con la implantación de la LOE (2006), fueron sustituidos por los Programas de Cualificación Profesional Inicial (PCPI). Estos últimos itinerarios educativos permiten al alumnado desarrollar competencias y habilidades personales y laborales que facilitan su acceso al mercado de trabajo, gracias a la obtención de una titulación del Sistema Nacional de Cualificaciones y Formación Profesional de Nivel 1 y al título de Graduado en ESO.

Así, tal como reflejan Riaño-Galán, García-Ruiz, Rodríguez y Álvarez-Arregui (2016), los PCPI se presentan como una de las estrategias fundamentales para la atención a la diversidad dirigidas hacia la consecución del éxito escolar por parte del alumnado y, además, desafiando el riesgo de su exclusión social y educativa. No obstante, desde la publicación de la LOMCE (2013), estos itinerarios adquieren la denominación de ciclos de Formación Profesional Básica (en adelante FPB), teniendo un mayor peso en ellos el desarrollo de materias instrumentales, y otorgándose únicamente la titulación relativa a la Cualificación y Formación Profesional (salvo el alumnado que cumpla las excepciones recogidas en la disposición transitoria del RD 1058/2015, de 20 de noviembre, que sí podrán obtener también el título de Graduado en ESO). De esta manera, esta nueva situación supone un paso atrás hacia el tránsito a la vida adulta de los alumnos con DFI desde una perspectiva inclusiva.

Más allá de la cuestión legislativa, debemos considerar el papel clave que desempeña el docente de este tipo de itinerarios formativos como agente mediador de la inclusión socio-laboral, ya que cuenta con un lugar privilegiado para mostrar las potencialidades de estos jóvenes como trabajadores en el contexto social y empresarial, contribuyendo a impulsar su posterior inserción socio-laboral (Negri y Leiva, 
2016). Para ello, es preciso que este cuente con los apoyos institucionales, sociales y materiales que facilitarán el establecimiento de relaciones cercanas y directas con otros agentes socioeducativos.

Por tanto, el estudio de investigación que presentamos a continuación tiene como finalidad destacar el liderazgo de los docentes de FPB en su función de mediadores para la inclusión a nivel social y laboral de los jóvenes con DFI.

\subsection{Liderazgo docente para la mediación entre el contexto de los ciclos de FPB y el mercado laboral}

Para la adquisición de los aprendizajes propios de la formación profesional de los jóvenes con DFI, se hace imprescindible destacar la importancia del desarrollo y potenciación del liderazgo de los docentes de los citados itinerarios formativos. Para ello, debemos considerar el carácter transformador del mismo tanto en los centros educativos, como en las sociedades y en los procesos de aprendizaje que experimenta el alumnado (Contreras, 2016).

En este sentido, diversos autores como Bernal e Ibarrola (2015), señalan la importancia del liderazgo transformacional, donde el docente se manifiesta como un elemento clave de la mejora y optimización de los procesos de enseñanza-aprendizaje. Por su parte, San Saturnino y Goicoechea (2012), en sus investigaciones sobre el liderazgo transformacional de los profesores en el aula, asocian positivamente este tipo de liderazgo con los comportamientos, percepciones y resultados de aprendizaje de los alumnos, lo cual deriva en un mayor empoderamiento de estos últimos. De esta manera, el alumno adquiere una mayor participación y autodeterminación dentro del aula, lo cual es especialmente beneficioso en el caso de los alumnos con DFI.

Esta cuestión resulta especialmente significativa, dado que este colectivo ha sido invisibilizado tanto en el currículum como en la organización y estructura de los centros educativos durante décadas, dado que hasta los años 70 estos alumnos eran escolarizados en centros segregados y no es hasta Ley 14/1970, de 4 de agosto, General de Educación y Financiamiento de la Reforma educativa, que se les permite el acceso a los centros ordinarios en aulas segregadas. Así pues, hasta la implantación de la LOE (2006) no se produce el acceso generalizado de estos jóvenes en el aula ordinaria del centro educativo. Igualmente, aún en la actualidad, aunque existen recursos y programas específicos para la atención a la diversidad de este alumnado, encontramos que la representación en el currículo educativo de las personas con discapacidad es prácticamente inexistente. Un ejemplo de ello podemos encontrarlo en el estudio de Moya-Mata, Ruiz, Martín, Pérez y Ros (2017), que en su estudio sobre la representación de estas personas en los libros de texto, encuentran que solo aparecen en un 0,62 \% de las imágenes.

No obstante, el liderazgo docente no puede limitarse al aula, sino que trasciende a todo el contexto educativo (Bernal e Ibarrola, 2015), produciéndose de esta manera un cambio en la cultura e ideología de los aprendizajes que suceden en el mismo y que tienen un carácter significativo y transformador.

Relacionando la importancia del liderazgo docente con la formación e inserción socio-laboral de los jóvenes a los que nos referimos, debemos prestar atención a las 
investigaciones de Pallisera, Vilà, Martín y Puyaltó (2014) sobre el proceso de transición escuela-empleo que experimentan estos jóvenes. De sus estudios subyace la idea de que no existe una coordinación entre los servicios que atienden a su capacitación profesional y orientación e inserción socio-laboral. Al mismo tiempo, se destaca la necesidad de liderazgo durante este proceso, el cual debería ser ejercido por parte de los agentes educativos de los centros de educación secundaria (Pallisera et al., 2014).

Contreras (2016) propone una serie de recomendaciones para la renovación de la escuela actual, confiriendo una especial trascendencia al liderazgo pedagógico. Para ello, establece la necesidad de demandar su participación en acciones como la toma de decisiones, la dirección de comunidades de aprendizaje, y la asignación de funciones para la mentoría y mediación entre diversos agentes de la comunidad educativa.

Nos centraremos por tanto en esta última propuesta, donde los docentes de los ciclos de FPB cuentan con un amplio potencial para desempeñar el rol de mediadores entre los contextos escolares y laborales facilitadores de la inserción en el mercado de trabajo de los jóvenes con DFI. Esto se debe al lugar privilegiado que estos ocupan dentro de los centros educativos, al tener la misión de promover la adquisición de una formación en habilidades para el empleo y la capacitación profesional por parte de estos jóvenes. Del mismo modo, de acuerdo con Pereda, De Prada y Actis (2003), estos profesionales también gozan de una posición de mediadores entre las políticas legislativas, la actividad económica y el contexto familiar, lo cual les ayuda a conocer fielmente la realidad en la que estos se desenvuelven, siendo conscientes de sus potencialidades y limitaciones reales.

La necesidad de la presencia de una figura que actúe como mediador durante los procesos de inserción laboral de los jóvenes con DFI, se sustenta además en los supuestos de aplicación de la mediación en discapacidad que proporciona Munuera (2013), entre los que destaca la discriminación en el empleo por motivo de discapacidad: falta de adaptación funcional para el adecuado desempeño del trabajo, es decir, igualdad de oportunidades con las prestaciones adecuadas. En este caso, debemos recalcar las desventajas sociales que existen en la actualidad para que se produzca la incorporación de estos jóvenes al mercado laboral que, unidas a sus necesidades de apoyo, requieren de una profunda concienciación y sensibilización por parte del ámbito empresarial y de la sociedad en general.

Por lo tanto, en los procesos de inclusión socio-laboral de los jóvenes con DFI, debemos trascender los muros de la intermediación laboral, apostando por la mediación. Cuando la intermediación laboral se encarga de conciliar, orientar y gestionar la información en materia laboral de sus entidades (Munuera, 2016), la mediación tiene como elemento fundamental el diálogo y la comunicación entre las partes implicadas a través de la intervención de un tercero (Munuera, 2016; Iglesias y Medina, 2017). En el caso del acceso al empleo del alumnado con DFI de los ciclos de FPB, la implicación de los docentes como mediadores resulta esencial. Estos profesionales comparten varias horas semanales con este alumnado y conocen de cerca sus habilidades, potencialidades y expectativas. Esta cuestión, unida a las habilidades del orientador en sus funciones de orientación y asesoramiento, y al conocimiento de este sobre las demandas del mercado laboral, resulta clave en un proceso de mediación laboral de éxito. 
Este proceso resulta además enriquecedor para el sujeto susceptible de acceder al mercado laboral, al otorgarle autonomía y capacidad de decisión en la gestión del conflicto, fortaleciendo así su autodeterminación. En relación a esta idea, la autodeterminación debe jugar un papel fundamental en la formación para el empleo de los jóvenes con DFI y su posterior inclusión socio-laboral. No obstante, estudios realizados por Pallisera et al. (2014) ponen de manifiesto que los profesionales de los ámbitos escolar y postescolar están de acuerdo en señalar que en los centros de educación secundaria no suele tenerse en cuenta el aprendizaje de contenidos relacionados con la autodeterminación del individuo, centrándose las intervenciones y adaptaciones curriculares realizadas a estos jóvenes en aspectos académicos y curriculares.

Por lo tanto, es necesario que consideremos la existencia de ciertas insuficiencias que deben ser superadas si queremos que el docente ocupe un papel de liderazgo en el proceso de mediación que subyace al tránsito desde la escuela al empleo. Estas insuficiencias se encuentran relacionadas con la organización y gestión de los centros educativos, así como con las funciones que les han sido asignadas a los docentes desde las instituciones competentes.

\section{Método}

\subsection{Objetivos}

Este estudio de investigación tiene como finalidad indagar en la formación e inserción socio-laboral de los jóvenes con DFI que realizan sus estudios de FPB, donde el docente debe ocupar un papel de mediador, partiendo del liderazgo que se le otorga desde el centro educativo en la consecución de estos procesos. De esta manera, hemos establecido los siguientes objetivos: a) Conocer las funciones a desempeñar por los docentes de FPB que faciliten la inserción laboral de estos jóvenes; b) Identificar las competencias sociales y personales claves a considerar por los docentes para la capacitación socio-laboral de los jóvenes con DFI y c) Analizar el trabajo interdisciplinar de los docentes de FPB como herramienta que favorece el liderazgo de estos profesionales en los procesos de inserción socio-laboral del alumnado con DFI.

\subsection{Diseño de investigación}

Hemos optado por la realización de un modelo de investigación mixto, que nos permite llevar a cabo la triangulación de los datos obtenidos, llegando a alcanzar el máximo grado de reconocimiento posible de una misma realidad desde dos metodologías de aproximación diferenciadas (Sánchez, 2015).

En la parte cualitativa de la investigación nos decantamos por una estrategia de corte holístico, descriptivo e interpretativo, utilizando para ello una metodología de estudio de casos, que nos va a permitir describir, comprender y analizar las situaciones que acontecen en la realidad objeto de nuestro estudio. Esto nos permitirá conocer la realidad general del contexto seleccionado, estableciendo relaciones entre las variables que acontecen dentro del mismo. Así pues, el estudio de casos permite 
la transferencia de la información obtenida hacia la teoría, como señalan Álvarez y San Fabián (2012).

\subsection{Contexto y participantes}

El contexto seleccionado para la recogida de información en la vertiente cualitativa de este estudio se centra en la realidad educativa y formativa-laboral de los ciclos de FPB del IES Concepción Villalba. Se trata de un centro de Educación Secundaria, que cuenta con numerosos recursos destinados a atender a la diversidad de todo su alumnado, resultando ser un referente en la capacitación socio-laboral del alumnado con DFI en la provincia de Málaga. Desde el centro educativo, se ofertan tres itinerarios de PFB, con las especialidades de cocina, jardinería y marroquinería, formándose de 8 a 10 alumnos de entre 18 y 23 años ${ }^{1}$ en cada uno de estos programas.

Desde este contexto, pudimos llevar a cabo un total de nueve entrevistas a diferentes participantes: tres profesores de taller de cada una de las especialidades de FPB, una de las dos orientadoras del centro educativo, una de las profesoras de apoyo de Pedagogía Terapéutica, dos alumnas que se encuentran formándose en los itinerarios de FPB y sus correspondientes familias.

En la vertiente cuantitativa de este estudio de investigación, realizamos una recogida de información a un total de 38 participantes, siendo todos ellos profesionales del ámbito educativo que realizan su labor en un total de seis centros de Educación Secundaria de la provincia de Málaga. Los centros educativos fueron seleccionados con el requisito fundamental de atender desde los mismos a la diversidad del alumnado que presenta DFI, y desde los cuales se presta especial atención a su capacitación socio-laboral.

Así, contamos con la participación de un 36,8 \% de docentes de FPB, un 28,9\% de profesionales de apoyo a la integración, un 18,4 \% de docentes de ESO, un 13,2 \% de orientadores educativos y un $2,6 \%$ de profesionales educativos que pertenecen a otras especialidades.

\subsection{Procedimiento}

En la vertiente cuantitativa de este estudio, para la realización de la recolección de datos, elaboramos un cuestionario dirigido a los profesionales de diferentes centros de Educación Secundaria de la provincia de Málaga. Este cuestionario está formado por 30 preguntas tipo Likert, acompañados de una escala de opciones de respuesta par. El cuestionario está dividido en dos partes: una primera parte formada por 6

1. Los alumnos accedieron a estos programas cuando todavía eran itinerarios de PCPI, por lo que de acuerdo con el Art. 5.1. de la Orden ECl/2755/2007, de 31 de julio, por la que se regulan los programas de cualificación profesional inicial que se desarrollen en el ámbito de gestión del Ministerio de Educación y Ciencia, podían tener en el momento de su incorporación edades entre los 16 y los 21 años. Dadas sus características personales, son alumnos con una amplia trayectoria de fracaso escolar que han repetido varios cursos, tanto en ESO como en estos itinerarios. 
cuestiones, que se centran en conocer los datos personales y profesionales de los participantes; y una segunda parte, constituida por 24 preguntas dirigidas a conocer las opiniones sobre los aspectos educativos y formativos que estos manifiestan respecto a la realidad en la que se desenvuelven.

El cuestionario se configuró a partir de las propuestas de un grupo de expertos sobre el ámbito. Tras obtener una versión inicial, dicho grupo de expertos debatió sobre la validez teórica del instrumento. Tras dos reuniones de hora y media cada una de ellas, se llegó a un acuerdo que constituye el instrumento utilizado en esta investigación.

Por su parte, en la parte cualitativa de la investigación se desarrollaron un total de 5 entrevistas a los profesionales que intervienen con los alumnos con DFI en los ciclos de FPB del centro objeto de nuestro estudio de caso. De esta manera, conocimos las percepciones de dos miembros del Departamento de Orientación y de tres profesores de los Módulos asociados a unidades de competencia del Catálogo Nacional de Cualificaciones Profesionales, o lo que es lo mismo, los profesores de taller de cada especialidad.

Optamos por la elaboración de entrevistas semiestructuradas, contando con un guion de preguntas abiertas, y considerando que en esta estrategia de recogida de información podrían surgir temáticas emergentes en el transcurso de estas. Las entrevistas se realizaron de manera individual con cada profesional y tuvieron una duración de entre 40 y 60 minutos cada una.

Se llevó a cabo un análisis descriptivo básico de datos de la escala. Igualmente, se llevó a cabo un análisis de contenido de las entrevistas estableciendo un sistema de categorías para todas ellas. La validez de los resultados se aseguró a través de un procedimiento de triangulación de instrumentos. Para los análisis se utilizaron el SPSS versión 24 y el NUDIST 8.0.

\section{Resultados}

\subsection{Sobre la labor docente}

En primer lugar, hemos indagado en los aspectos relativos a la labor que desempeñan los docentes de los centros educativos para afrontar la formación e inserción socio-laboral de los jóvenes con DFI. En este sentido, hemos preguntado a estos profesionales si el trato que reciben los alumnos con DFI por parte de sus profesores es igual que el del resto de sus compañeros. Así, una mayoría de un 60,50 \% de los profesionales participantes en este estudio, indica que sí, y en algunos casos se les ofrecen más apoyos y ayudas que a sus compañeros. El resto se decanta por las demás opciones, existiendo un 15,80 \% de profesionales que reconoce que no, porque los profesores no tienen tiempo de atenderles como necesitan, un 13,20\% que afirma que sí, totalmente, y un 10,50 \% que determina que no, algunos profesionales delegan la educación de estos alumnos en el personal de apoyo del centro (profesores de apoyo de Pedagogía Terapéutica y Audición y Lenguaje, logopeda, orientador, etc.). 


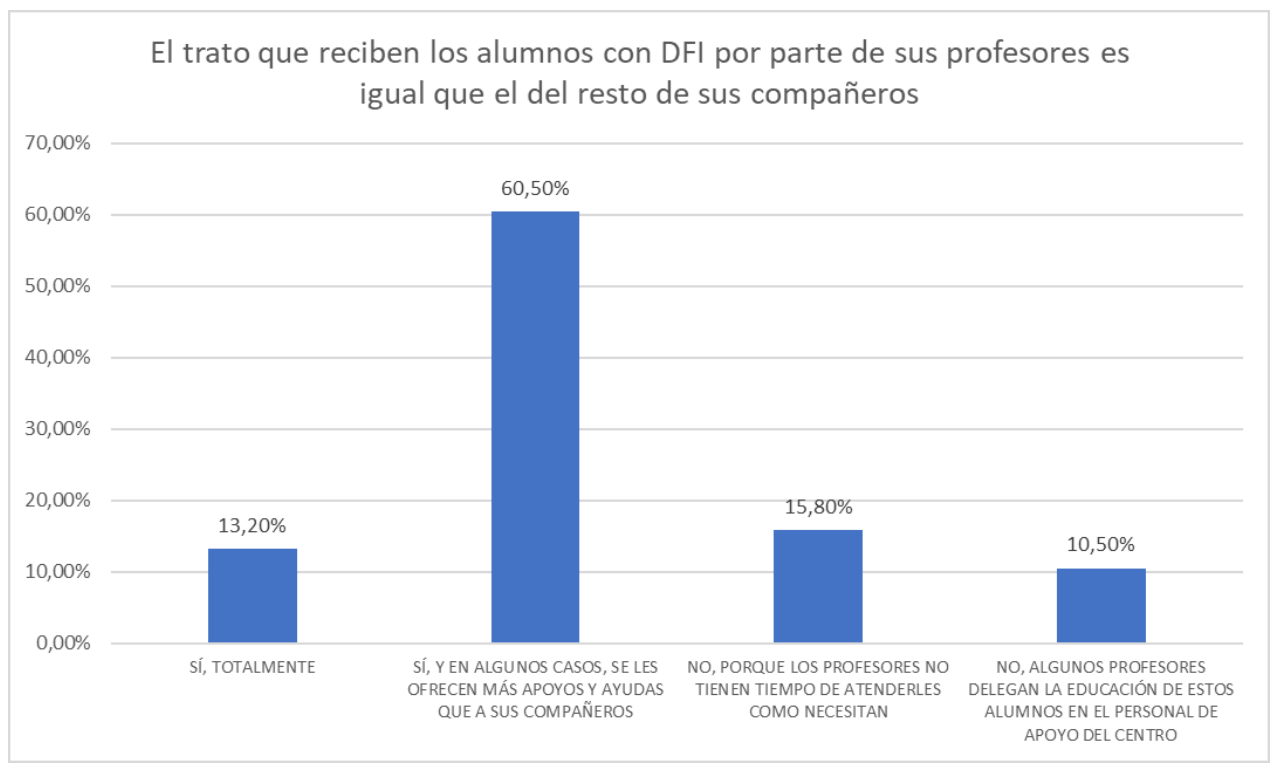

Gráfico 1. El trato que reciben los alumnos con Diversidad Funcional Intelectual por parte de sus profesores es igual que el del resto de sus compañeros

Fijándonos en los datos obtenidos, tenemos claro los participantes de nuestro estudio consideran que estos alumnos gozan de un trato respetuoso, justo e igualitario por parte de sus profesores, determinando que en la mayoría de las ocasiones cuentan con una serie de apoyos específicos para abordar de manera comprensiva su desarrollo educativo. Podemos extraer entonces la idea de que el profesorado se encuentra concienciado acerca de las necesidades individuales que presenta este alumnado para alcanzar con éxito su desarrollo educativo. Y es que para que los alumnos con DFI puedan para alcanzar el éxito personal, laboral y social, resultan determinantes las actitudes y concepciones socioeducativas que presenta el profesorado hacia sus características y necesidades individuales.

No obstante, los apoyos proporcionados por el profesorado suelen centrarse en la adaptación de actividades curriculares o la intervención individualizada de profesionales de apoyo a la integración; estrategias más centradas en potenciar el crecimiento académico del alumno o favorecer su comunicación directa con el entorno que le rodea, que en fomentar habilidades y destrezas de índole socio-laboral.

De forma complementaria a las ideas previamente formuladas, hemos querido además indagar en cuáles son las funciones que desempeñan los profesionales educativos que en ocasiones se encuentran alejadas de sus labores puramente docentes y que suponen un valor añadido en el aprendizaje de habilidades y competencias laborales por parte de los jóvenes. Hablamos, por ejemplo, de la localización de empresas en las que los estudiantes puedan realizar sus prácticas profesionales para finalizar con éxito su proceso de capacitación profesional: 
Yo hago un esfuerzo también porque me tengo que multiplicar, tengo que hablar con el empresario, tengo que coger mi coche, tengo que salir, tengo que hacer el seguimiento, y ahora mismo lo único que nos hacen a los profesores es meternos más horas, quitarnos dinero, entonces, la gente está quemada, se junta todo. Si no hay dinero, no hay alegría, todo se complica y por supuesto cosas sociales mucho más, así que así estamos. (Entrevista a profesor de taller de FPB Cocina).

La labor del docente de FPB actualmente no se centra únicamente en la impartición de determinadas materias, sino que además debe realizar una serie de actividades complementarias que favorezcan el desarrollo integral de los jóvenes de cara a su inserción en el mercado de trabajo. En este sentido, podemos decir que los docentes actúan como mediadores entre el centro educativo y el ámbito laboral, llevando a cabo actuaciones de información, concienciación y sensibilización que favorezcan la concepción social que existe actualmente en el mercado empresarial acerca de este colectivo.

Podemos observar una alta implicación por parte del docente entrevistado, que se extralimita de sus labores puramente académicas para contribuir a mejorar el futuro socio-profesional de sus alumnos. Lamentablemente, este tipo de tareas de gran valor no están reconocidas por las administraciones competentes como actividades que formen parte del campo de actuación de estos profesionales, con su consecuente falta de reconocimiento por parte de las mismas. Así, muchos profesionales se limitan a realizar únicamente las labores asignadas, mientras otros pueden sentirse frustrados o sobrecargados por la alta responsabilidad que recae sobre ellos, por lo que, en la mayoría de los casos podrían optar por dejar de lado este tipo de actividades dirigidas al diálogo y la negociación con el mercado empresarial.

A tal efecto, sería conveniente fomentar la colaboración de los centros educativos con entidades del Tercer Sector que luchen por la inclusión socio-laboral de este colectivo, estableciendo una clara continuidad entre la capacitación y la inclusión laboral de estos.

\subsection{Sobre la selección de los aprendizajes}

En segundo lugar, hemos indagado en cuáles deberían ser las habilidades a fomentar por los docentes para atender a la educación integral del alumnado, y que resultan de gran importancia para favorecer la capacitación de los jóvenes con DFI hacia el acceso al empleo y la vida adulta.

En este sentido, un $44.40 \%$ de los docentes señala la relevancia de la resolución de problemas, seguido de un 33,30 \% que apuesta por la capacidad de aprender a aprender. Al mismo tiempo, un 11,10\% de los participantes destaca la importancia de la capacidad de tomar sus propias decisiones, un 7,40 \% determina la autoestima y un 3,70 \% considera relevante la capacidad de organización de la información. 


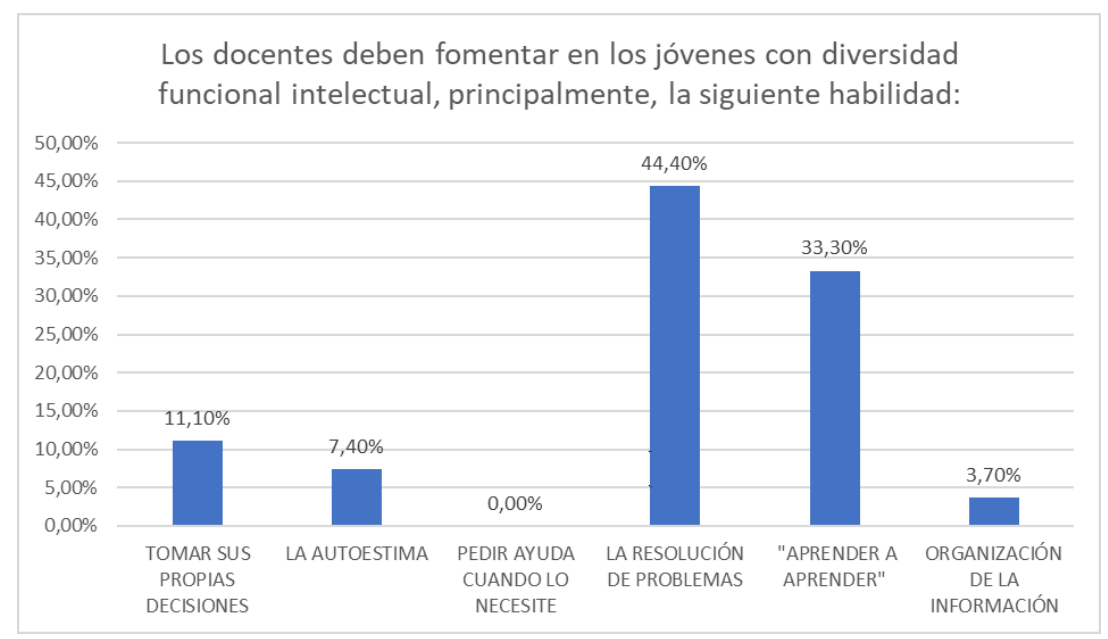

Gráfico 2. Los docentes deben fomentar en los jóvenes con diversidad funcional intelectual, principalmente, la siguiente habilidad

Fijándonos en los datos obtenidos, los docentes deben desempeñar un rol esencial en el desarrollo de competencias personales del alumnado, y resulta significativo el hecho de que son ellos mismos quienes consideran y priorizan en la profundización del aprendizaje de aquellas de mayor relevancia para el acceso a la vida adulta. Como podemos observar, tanto la resolución de problemas como la capacidad de aprender a aprender, son las competencias mejor valoradas por una gran mayoría de los docentes para que estos jóvenes puedan acceder con éxito a los contextos propios de la vida adulta. Además, podemos decir que se trata de dos variables relacionadas con el desarrollo autónomo del individuo y su autodeterminación, que a su vez contribuirán a mejorar sus habilidades comunicativas y de gestión de conflictos.

En resumen, podríamos decir que los docentes apuestan por el desarrollo de habilidades transferibles tanto al mercado de trabajo como al resto de contextos propios de la vida adulta, manifestando estos la relevancia de dotar al alumno de aprendizajes significativos que trasciendan más allá de los muros de los centros educativos.

En relación con la temática anteriormente expuesta, hemos querido profundizar sobre cuáles son las estrategias de aprendizaje y los mecanismos de apoyo que el profesorado pone en práctica en el aula para atender a las necesidades individuales que presentan estos jóvenes:

Profesora: Yo prefiero ese módulo sustituirlo por uno en el que el alumno adquiera unas destrezas que le permitan en un futuro conseguir un trabajo, que es de lo que se trata. Eso ya es flexible dentro de mi aula.

Entrevistadora: Te vas adaptando a cada uno...

Profesora: Exactamente, aunque en el currículum es diferente pero ya te adaptas tú como profesional.

Entrevistadora: Vas potenciando las habilidades que ves que tienen. 
Profesora: Claro, tú ves las necesidades y actúas sobre eso. Quizá en los contenidos teóricos, pues en la práctica sabes ya más o menos cómo tienes que organizar las clases. (Entrevista a profesora de taller FPB Marroquinería).

Como podemos observar, esta docente tiene muy presente la necesidad de impulsar desde el aula una dinámica de enseñanza-aprendizaje que resulte significativa para todo el alumnado, seleccionando contenidos y habilidades que les puedan ser de utilidad para el desarrollo de sus actividades cotidianas.

Para ello, en lugar de ceñirse a los contenidos que se han establecido previamente en el currículum desde un enfoque rígido y globalizador, la docente apuesta por otorgar un enfoque constructivista de la educación, que considere el nivel de competencia cognitiva, social y laboral ya adquirida por el alumno. De esta manera, se establece una continuidad entre los aprendizajes que suceden dentro del aula, y la adquisición y el afianzamiento de los mismos resultan más efectivos, al no ser ajenos a la realidad que les rodea.

Así, la transferibilidad de los aprendizajes desde el contexto educativo hacia las actividades de la vida diaria, surge de forma natural y espontánea, sin tener que recurrir a procesos más complejos de razonamiento y relación de ideas que se muestran alejadas unas de otras.

\subsection{Sobre el trabajo interdisciplinar}

Por último, hemos preguntado si los profesionales de los centros educativos colaboran interdisciplinariamente para ofrecer una educación de calidad a los jóvenes con DFI. En esta línea, hemos sido conscientes de que un 61,10 \% de ellos considera que sí, en algunas ocasiones piden asesoramiento al personal de apoyo (logopeda, profesor de apoyo de Pedagogía Terapéutica o Audición y Lenguaje, orientador, etc.), mientras un $27,80 \%$ señala que sí siempre, seguidos de un $8,30 \%$ que indica que no, pues resulta imposible por cuestiones de tiempo, espacio, etc. y de un 2,80\% que manifiesta que no, cada profesional se limita a impartir su área de aprendizaje.

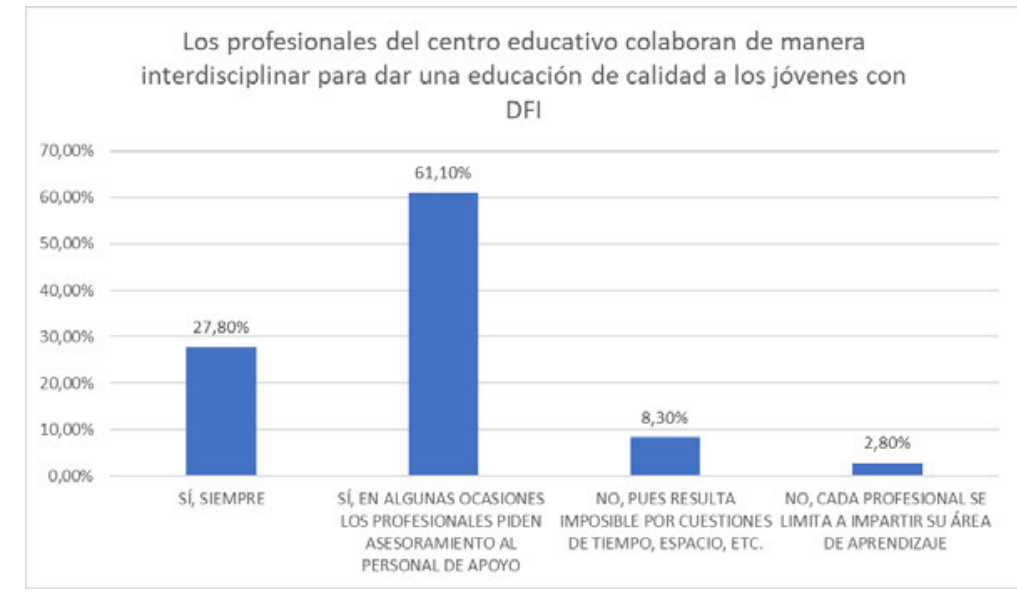

Gráfico 3. Los profesionales del centro educativo colaboran de manera interdisciplinar para dar una educación de calidad a los jóvenes con DFI 
Como podemos observar, la mayoría de los docentes suele apostar por este tipo de intervención educativa, favoreciendo así el aprendizaje integral del alumnado con DFI. Y es que, en la atención educativa de estos jóvenes, resulta fundamental que los aprendizajes adquiridos en la escuela puedan ser aplicables en el contexto socio-laboral.

Apostar por un cambio estructural, metodológico y organizativo en las dinámicas que se establecen dentro del aula resulta esencial para favorecer el trabajo colaborativo del profesorado. Sin embargo, en muchas ocasiones, los docentes no cuentan con una formación inicial y permanente capaz de ajustarse a estas premisas, y además no disponen de los recursos materiales y humanos suficientes para atender a los apoyos concretos que necesitan los alumnos con DFI. Al mismo tiempo, sería necesario establecer espacios y tiempos para el diálogo e intercambio de la práctica docente, que permita implantar una transformación real en el desarrollo de la docencia desde una perspectiva global e integradora de los aprendizajes.

Por otra parte, hemos preguntado al profesorado si considera suficientes los recursos humanos especializados que dispone para atender a la diversidad de sus alumnos en los ciclos de FPB:

Tengo una profesora de $\mathrm{PT}^{2}$ que viene una serie de horas a la semana, quizá sería más conveniente que viniera más horas porque es un grupito muy heterogéneo, con muchas necesidades por la discapacidad tan grande que tienen los alumnos. Quizá que estuviera más tiempo conmigo sí me vendría bien. Tengo varios alumnos que no pueden comunicarse, normalmente ellos me entienden pero no pueden hablar, entonces tengo intérpretes de lengua de signos que vienen una serie de horas a la semana y me ayudan también. (Entrevista a profesora de taller de FPB Jardinería).

La docente considera insuficientes las intervenciones que realizan la profesora de apoyo de Pedagogía Terapéutica y los intérpretes de lengua de signos dentro de su aula, debido a la gran heterogeneidad del grupo de alumnos al que atiende. A pesar de tratarse de grupos reducidos, este alumnado necesita una atención personalizada y una serie de apoyos individuales que les permitan seguir el desarrollo de las clases y relacionarse adecuadamente con sus compañeros y profesora.

A este efecto, resulta fundamental destacar el papel que desempeña el personal de apoyo especializado, dada su cualificación en la atención a las particularidades de todo el alumnado, en base a sus capacidades y necesidades individuales.

No obstante, la intervención educativa de los jóvenes con DFI no puede depender exclusivamente de la presencia o no de estos profesionales dentro del aula ordinaria, sino que los apoyos y atenciones individualizadas deben ser transversales a todas las

2. La docente se refiere coloquialmente a la profesora de apoyo de Pedagogía Terapéutica del centro educativo. 
actividades que se desarrollen dentro del contexto del centro educativo. Para ello, resulta fundamental el diálogo entre todos los docentes que intervienen en el desarrollo integral del alumno, cobrando una especial importancia los profesionales especializados para atender a sus necesidades específicas. Igualmente, se requiere del trabajo cooperativo e interdisciplinar del profesorado, donde a través de un liderazgo compartido, así como del intercambio de inquietudes, experiencias y estrategias metodológicas, se atienda a las necesidades individuales de estos jóvenes dentro y fuera del aula.

Hablamos por tanto, de modelos de intervención educativa basados en la aplicación de una metodología de investigación-acción de los docentes, que permita transformar los procedimientos, contextos y paradigmas de atención a la diversidad que se encuentran presentes en la escuela.

\section{Discusión y CONCLUSIONES}

En la línea de estudios anteriores (Pallisera, Fullana, Puyaltó y Vilà, 2016; Pallisera et al., 2018), resulta clave el incremento de los tiempos y espacios de coordinación interinstitucional y del propio liderazgo pedagógico de los docentes de FPB. La formación de los jóvenes con DFI requiere una redefinición organizativa y curricular lo más ajustada y personalizada posible en relación con sus características y singularidades.

En este sentido, desde los centros educativos resulta esencial el ejercicio práctico y efectivo de un liderazgo compartido que permita atender a la diversidad de todo el alumnado mediante el trabajo interdisciplinar del profesorado. Gracias a esta metodología de trabajo interdisciplinar, los docentes pueden resolver sus inquietudes, problemas e incidencias dentro del aula apoyándose en la labor profesional y la formación de otros docentes, produciéndose así una optimización de los recursos humanos existentes dentro del centro educativo y con una nítida apertura a la sociedad y al empleo.

Esta estrategia de intervención va a permitir al alumnado desarrollar una mayor capacidad de transferencia de los aprendizajes, permitiéndole abordar los desafíos presentes en la sociedad actual (Serrato, González y Olmos, 2016), potenciando en ellos habilidades sociales que faciliten su adaptación al contexto laboral y actitudes de responsabilidad y autonomía personal (Marín, García y Sola, 2013).

La problemática en la utilización de un modelo de trabajo interdisciplinar por parte de los docentes de FPB reside en la situación de excesiva burocratización de las tareas formativas y de gestión pedagógica que pueden ocasionar un malestar o sobrecarga emocional en los docentes. Esto puede tener implicaciones negativas para la adecuada personalización y orientación de los aprendizajes del alumnado con DFI.

De acuerdo con Castillo y Canul (2015), el papel del docente como mediador es el de erigirse como puente entre el alumnado y los procesos y contextos donde éste se desenvuelve, partiendo de su propia realidad y ayudándole a ser el constructor de su propio aprendizaje. Con el objetivo de alcanzar la inclusión de los jóvenes con DFI en el mercado de trabajo, el papel de los docentes es el de acompañante y 
mediador, teniendo un papel esencial en la concienciación del mercado empresarial. Esta acción facilitará una mayor apertura y flexibilidad en los procesos económicos y laborales, que resultarán más receptivos para dar respuesta a las necesidades de participación y visibilización que tiene actualmente el colectivo.

Todo este proceso de transformación de la labor docente requiere de una capacitación adecuada del profesorado, así como de una implicación real de este hacia las necesidades concretas de los jóvenes con DFI en su inclusión social y laboral. Ni que decir tiene que la voz de los jóvenes con DFI debe ser atendida porque es un elemento clave para construir una formación pedagógica de calidad y en inclusión (Noell et al., 2018).

En definitiva, el impulso del liderazgo de los docentes como agentes de mediación en la inclusión socio-laboral provocará la apertura del centro educativo hacia otras instancias sociales igualmente responsables de la inclusión social de este colectivo, emergiendo así nuevas posibilidades para la inserción laboral de los jóvenes con DFI.

\section{REFERENCIAS BIBLIOGRÁFICAS}

Álvarez, C. y San Fabián, J. L. (2012). La elección del estudio de caso en investigación educativa. Gazeta de Antropología, 28(1). Recuperado de http://hdl.handle. net/10481/20644.

Bernal, A. e Ibarrola, S. (2015). Liderazgo del profesor: objetivo básico de la gestión educativa. Revista Iberoamericana de Educación, 67, 55-70. Recuperado de https://rieoei.org/historico/documentos/rie67a03.pdf.

Castillo, S. E. y Canul, P. Y. (2016). Docente mediador en la formación de competencias en educación básica. Revista Iberoamericana en la formación de competencias en educación básica, 2(4). Recuperado de http://www.pag.org.mx/index.php/ PAG/article/view/334.

Contreras, T. S. (2016). Liderazgo pedagógico, liderazgo docente y su papel en la mejora de la escuela: una aproximación teórica. Propósitos y Representaciones, 4(2), 231-284. Recuperado de https://www.mendeley.com/catalogue/liderazgopedag\%C3\%B3gico-liderazgo-docente-y-su-papel-en-la-mejora-la-escuela-unaaproximaci\%C3\%B3n-te\%C3\%B3rica/.

Fundación ONCE. (2015). Empleo y Formación. Recuperado de: http://www. fundaciononce.es/es/pagina/empleo-y-formacion.

Iglesias, E. y Medina, E. (2017). Oportunidad de la mediación en la intervención social con personas en situación de discapacidad. Revista de mediación, 10(1), 14. 20. Recuperado de https://revistademediacion.com/articulos/oportunidad-lamediacion-la-intervencion-social-personas-situacion-discapacidad/.

García-Gómez, S., Ordoñez-Sierra, R. e Izquierdo, R. (2018). Conocer a las familias del alumnado de formación profesional, un reto para la investigación educativa. Contextos educativos, 22, 149-163. http://doi.org/10.18172/con.3113. 
Ley 14/1970, de 4 de agosto, General de Educación y Financiamiento de la Reforma Educativa, Boletín Oficial del Estado, 187, de 6 de agosto de 1970, 12525-12546. Recuperado de https://www.boe.es/buscar/doc.php?id=BOE-A-1970-852.

Ley Orgánica 2/2006, de 3 de mayo, de Educación. Boletín Oficial del Estado, 106, 4 de mayo de 2006, 1-110. Recuperado de https://www.boe.es/buscar/pdf/2006/ BOE-A-2006-7899-consolidado.pdf.

Ley Orgánica 8/2013, de 9 de diciembre, para la mejora de la calidad educativa. Boletín Oficial del Estado, 295, de 10 de diciembre de 2013, 97858-97921. Recuperado de https://www.boe.es/boe/dias/2013/12/10/pdfs/BOE-A-2013-12886.pdf.

Marín, J. A., García, M. y Sola, J. M. (2013). Reflexión y análisis sobre los programas de Cualificación Profesional Inicial como medida de inclusión social y educativa en Andalucía (España). REICE. Revista Iberoamericana sobre Calidad, Eficacia y Cambio en Educación, 12(1), 83-102. Recuperado de https://revistas.uam.es/ index.php/reice/article/viewFile/2866/3083.

Moya-Mata, I., Ruiz, L, Martín, J., Pérez, P. y Ros, C. (2017). La representación de la discapacidad en las imágenes de los libros de texto de Educación Física: ¿inclusión o exclusión? Retos, 22(2), 88-95. Recuperado de https://recyt.fecyt.es/index. php/retos/article/view/52191.

Munuera, M. P. (2013). Mediación con personas con discapacidad: igualdad de oportunidades y accesibilidad de la justicia. Revista Política y Sociedad, 50, 163-178. Recuperado de https://eprints.ucm.es/20504/1/39349-57475-1-PB.pdf.

Munuera, M. P. (2016). Inclusión sociolaboral de las personas con discapacidad. Mediación laboral. Mediaciones Sociales, 14, 161-179. http://doi.org/10.5209/ rev_MESO.2015.n14.51564.

Negri, M. I. y Leiva, J. (2016). El papel de la formación profesional en la inclusión socio-laboral de jóvenes con Diversidad Funcional Intelectual. Revista nacional e internacional de Educación Inclusiva, 9(3), 13-28. Recuperado de https://dialnet.unirioja.es/servlet/ articulo?codigo $=4867479$.

Negri, M. I. y Leiva, J. (2017). Los Profesionales Educativos como Agentes de Capacitación Socio-Laboral de los Jóvenes con Diversidad Funcional Intelectual. Revista Digital de Investigación en Docencia Universitaria, 11(1), 62-81. http://dx.doi.org/10.19083/ridu.11.518.

Noell, J. F., Pallisera, M., Osete, E. C., Rovira, C. P., Vilà, M. y Díaz-Garolera, G. (2018). Participando en investigaciones inclusivas. Un curso de formación en investigación desde el punto de vista de los participantes con discapacidad intelectual. Revista de Educación Inclusiva, 11(1), 193-210. Recuperado de http://www. revistaeducacioninclusiva.es/index.php/REl/article/view/336.

Pallisera, M., Fullana, J., Vilà, M., Jiménez, P., Castro, M., Puyaltó, C., Montero, M. y Martín, R. (2014). Análisis de los apoyos que reciben los jóvenes con discapacidad intelectual en su transición a la vida adulta en España: una investigación a partir de experiencias de profesionales y personas con discapacidad, Revista Española de Discapacidad, 2(2), 27-43. http://doi.org/10.5569/2340-5104.02.02.02. 
Pallisera, M., Vilà, M., Fullana, J., Martín, R. y Puyaltó, C. (2014). ¿Continuidad o fragmentación? Percepción de los profesionales sobre la coordinación entre servicios en los procesos de tránsito a la vida adulta de los jóvenes con discapacidad intelectual. Educatio Siglo XXI, 32(2), 213-232. Recuperado de http://revistas.um.es/ educatio/article/view/194161.

Pallisera, M., Fullana, J., Puyaltó, C. y Vilà, M. (2016). Changes and challenges in the transition to adulthood: views and experiences of Young people with learning disabilities and their families. European Journal of Special Needs Education, 31(3), 391-406. https://doi.org/10.1080/08856257.2016.1163014.

Pallisera, M., Vilà, M., Fullana, J., Díaz-Garolera, G., Puyalto, C. y Valls, M. J. (2018). The role of professionals in promoting independent living: Perspectives of selfadvocates and front-line managers. Journal of Applied Research in Intellectual Disabilities, 31(6), 1103-1112. http://dx.doi.org/10.1111/jar.12470.

Pereda, C., De Prada, M. A. y Actis, W. (2003). La inserción laboral de las personas con discapacidades. Revista del Ministerio de Trabajo y Asuntos Sociales, 55, 151 156. Recuperado de https://goo.gl/R8ZhkZ.

Riaño-Galán, A., García-Ruiz, R., Rodríguez, A. y Álvarez-Arregui, E. (2016). Calidad de vida e inserción socio-laboral de jóvenes con discapacidad. Revista Electrónica de Investigación Educativa, 18(1), 112-127. Recuperado de http://redie.uabc.mx/ redie/article/view/907.

Sánchez, M. C. (2015). La dicotomía cualitativo-cuantitativo: posibilidades de integración y diseños mixtos. Campo abierto. Monográfico, 11-30. Recuperado de https://mascvuex.unex.es/revistas/index.php/campoabierto/article/view/1679.

Serrate, S., González, M., Olmos, S. (2016). La acción socioeducativa interdisciplinar en la etapa de Educación Secundaria: situación y necesidades profesionales. Revista de Educación, 376, 200-228. https://doi.org/10.4438/1988-592XRE-2017-376-349.

Vilà, M., Pallisera, M. y Fullana, J. (2010). ¿Contribuye a la inclusión laboral la formación que recibe el alumnado con discapacidad intelectual en la ESO? Revista de Educación Inclusiva, 3(3), 51-66. Recuperado de https://goo.gl/7wFAcG. 